Mon. Not. R. Astron. Soc. 000, 1-14 (2017) Printed November 27, $2018 \quad$ (MN LTEX style file v2.2)

\title{
Intra-Night Optical monitoring of three $\gamma$-ray detected Narrow-line Seyfert 1 galaxies
}

\author{
Vineet Ojha ${ }^{1 \star}$, Gopal-Krishna ${ }^{1}$, Hum Chand ${ }^{1}$ \\ ${ }^{1}$ Aryabhatta Research Institute of Observational Sciences (ARIES), Manora Peak, Nainital - 263002, India
}

Accepted - Received —; in original form -

\begin{abstract}
For 3 radio-loud $\gamma$-ray detected Narrow-Line Seyfert 1 ( $\gamma$-ray NLSy1) galaxies, we report optical variability on intra-night and/or week-like time scales, based on five $\geqslant 3$ hours long monitoring sessions for each galaxy. The radio-loudness factors $\left(\mathrm{R}_{1.4 \mathrm{GHz}}\right)^{1}$ for these galaxies, namely $1 \mathrm{H} 0323+342(\mathrm{z}=0.0629)$, PKS J1222 $+0413(\mathrm{z}=0.966)$ and PKS J1505+0326 $(\mathrm{z}=0.408)$ are $\sim 318, \sim 1534$ and $\sim 3364$ at $1.4 \mathrm{GHz}$, respectively. For the most distant $\gamma$-ray NLSy1, PKS J1222+0413, Intra-Night Optical Variability (INOV) characterisation is presented for the first time. The blazar-like behaviour of the nearest $\gamma$-ray NLSy1 $1 \mathrm{H} 0323+342$, which showed strong INOV on 4 of the 5 nights, was unexpected in view of its recent reclassification as radio intermediate $\left(\mathrm{R}_{5 \mathrm{GHz}} \lesssim 25\right)$. Its particularly violent INOV is manifested by two optical outbursts lasting $\sim 1$ hour, whose rapid brightening phase is shown to imply a doubling time of $\sim 1$ hour for the optical synchrotron flux, after (conservatively) deducting the thermal optical emission contributed by the host galaxy and the Seyfert nucleus. A more realistic decontamination could well reduce substantially the flux doubling time, bringing it still closer in rapidity to the ultra-fast VHE $(>100 \mathrm{GeV})$ flares reported for the blazars PKS 1222+216 and PKS 2155-304. A large contamination by thermal optical emission may, in fact, be common for NLSy1s as they are high Eddington rate accretors. The present study further suggests that superluminal motion in the radio jet could be a robust diagnostic of INOV.
\end{abstract}

Key words: surveys galaxies: active galaxies: jets galaxies: photometry galaxies: Seyfert: general - $\gamma$-rays: individual $(1 \mathrm{H} 0323+342$, PKS J1222+0413 and PKS 1505+0326)

\section{INTRODUCTION}

Intensity variation over the entire accessible electromagnetic spectrum is one of the defining characteristics of active galactic nuclei (AGN). This trait is often utilised as an effective tool to probe their emission mechanism on physical scales that are inaccessible to direct imaging techniques (e.g., Wagner \& Witzel 1995; Urry \& Padovani 1995; Ulrich et al. 1997; Zensus 1997). The optical flux variations of AGN occurring on hour-like, or occasionally even shorter timescales are commonly known as Intra-Night Optical Variability (INOV) and it has come to be used quite extensively as a tracer of jet activity in blazars and other AGN classes (e.g., Miller et al. 1989; Gopal-Krishna et al. 1993, 1995; Jang \& Miller 1995; Heidt \& Wagner 1996; Bai et al. 1999; Romero et al. 1999; Fan et al. 2001; Stalin et al. 2004a;
Gupta \& Joshi 2005; Carini et al. 2007; Ramírez et al. 2009; Goyal et al. 2012, 2013a; Kumar et al. 2017).

In the case of blazars, the INOV phenomenon is usually associated with Doppler boosting of the jet's radiation, which not only amplifies any emission fluctuations occurring within the jet's plasma whose bulk relativistic motion is directed close to our line of sight but also shortens the timescales (e.g., Hughes et al. 1992; Marscher et al. 1992; Begelman et al. 2008; Ghisellini \& Tavecchio 2008; Giannios et al. 2009; Marscher 2014). At a subdued level, the same process may be at work in radio-quiet quasars (RQQs) due to the presence of a weak jet (Gopal-Krishna et al. 2003; Stalin et al. 2004a; Barvainis et al. 2005), although hot spots or flares on accretion discs may also be significant, if not the dominant contributor to their INOV (Mangalam \& Wiita 1993; Wiita 2006). For radio-quiet ${ }^{1} \mathrm{AGN}$ showing rapid X-ray

\footnotetext{
^ E-mail: vineet@aries.res.in
}

${ }^{1}$ Radio-loudness is usually parametrised by the ratio (R) of the rest- 
variability, such as Narrow-Line Seyfert 1 galaxies (NLSy1s), one may also expect to find short-term optical variations simply because the X-ray emission may have an optical tail. However, any such evidence is weak (e.g., Miller \& Noble 1996; Ferrara et al. 2001), although at longer time scales this phenomenon is well known (e.g., Rokaki et al. 1993; Gaskell 2006).

Fairly extensive information is now available on the INOV properties of luminous AGN for both radio-quiet and radio-loud varieties, including blazars, as summarized recently by Gopal-Krishna \& Wiita (2018). Much more scarce, however, are INOV data for their low-luminosity counterparts, e.g., NLSy1s (e.g., Kshama et al. 2017, and references therein). NLSy1s are characterised by the narrow width of optical Balmer emission lines FWHM( $\mathrm{H} \beta)<$ $2000 \mathrm{~km} \mathrm{~s}^{-1}$ (Osterbrock \& Pogge 1985; Goodrich et al. 1989; Pogge 2000; Sulentic et al. 2000), a small flux ratio $\left[\mathrm{O}_{I I I}\right]_{\lambda 5007} / H \beta<3$ (Shuder \& Osterbrock 1981). With some possible exceptions, they also exhibit strong [Fe VII] and [Fe X] lines (Pogge 2011), as well as strong permitted optical/UV Fe II emission lines (Boroson \& Green 1992; Grupe et al. 1999). As a class, NLSy1s are hosted by spiral galaxies (e.g., Crenshaw et al. 2003; Deo et al. 2006), although early-type galaxies have been considered as the host for the radio/ $\gamma$-ray loud subset of NLSy1s (Antón et al. 2008; León Tavares et al. 2014). Interestingly, their soft Xray emission which has a steep spectrum (Boller et al. 1996; Wang et al. 1996; Grupe et al. 1998), is often rapidly variable (Leighly 1999; Komossa \& Meerschweinchen 2000; Miller et al. 2000). At optical wavelengths, the first reports of rapid variability of NLSy1s on hour-like time scales appeared almost two decades ago (Miller et al. 2000; Ferrara et al. 2001; Klimek et al. 2004). A major boost to the studies of NLSy1 galaxies is likely to come from the recent publication of a large sample of 11,101 NLSy1s, out of which $\sim 600$ are radio loud (Rakshit et al. 2017, see also, Chen et al. 2018; Singh \& Chand 2018).

From the analysis of optical spectroscopic data, it has been inferred that the central black holes in NLSy1s have virial masses mostly in the range $10^{6}-10^{8} \mathrm{M}_{\odot}$ (e.g., Mathur 2000; Peterson et al. 2000; Yuan et al. 2008; Xu et al. 2012; Foschini et al. 2015; Cracco et al. 2016). Thus, they are typically 1-2 orders of magnitude less massive than the blackholes embedded in the cores of broad-line Seyfert galaxies and more powerful radio sources, like blazars and radioloud quasars, which are nearly always hosted by earlytype galaxies (e.g., McHardy et al. 1994; Boyce et al. 1998; Scarpa et al. 2000; Boroson 2002; Olguín-Iglesias et al. 2016), and have black-hole masses above $10^{8} M_{\odot}$ (e.g., Laor 2000; Dunlop et al. 2003; McLure \& Jarvis 2004; Chiaberge et al. 2005; Gopal-Krishna et al. 2008; Chiaberge \& Marconi 2011; Tadhunter 2016; Coziol et al. 2017). For $\gamma$-ray NLSy1s, somewhat higher $\mathrm{BH}$ masses (between a few $10^{7}$ and a few $10^{8} \mathrm{M}_{\odot}$ ) have been derived by modeling the optical/UV part of the SED in terms of a Shakura \& Sunyaev disc (e.g.,

frame flux densities at $5 \mathrm{GHz}$ and at $4400 \AA$, being $\mathrm{R} \leqslant$ and $>10$ for radio-quiet, radio-loud quasars, respectively (e.g., Kellermann et al. 1989). To differentiate the radio-loudness estimates based on the flux densities at $1.4 \mathrm{GHz}$ and $5 \mathrm{GHz}$, we have used $\mathrm{R}_{1.4 \mathrm{GHz}}$ and $\mathrm{R}_{5 \mathrm{GHz}}$ notations, respectively.
Doi et al. 2012; Calderone et al. 2013; Foschini et al. 2015; D'Ammando et al. 2016a; Paliya \& Stalin 2016) and they lie at the lower end of the BH masses of quasars/blazars. Physical scenarios for the possibility that virial masses of NLSy1s black holes may have been underestimated, include the effect of radiation pressure (Marconi et al. 2008), and a pole-on view of a disk-like BLR (e.g., Nagao et al. 2001; Bian \& Zhao 2004; Decarli et al. 2008; Shen \& Ho 2014; Baldi et al. 2016; see, however, Jarvela et al. 2017).

The existence of the above mass discrepancy came into the spotlight following the discovery of $\gamma$-ray emission from a few NLSy1s, using the Fermi/LAT ${ }^{2}$ (Abdo et al. 2009a,b, 2010; Calderone et al. 2011; Foschini et al. 2011; D'Ammando et al. 2012, 2015b; Yao et al. 2015; Paliya et al. 2018). Nearly 20 NLSy1 galaxies have since been catalogued as $\gamma$-ray emitters and all of them are also radio detected (e.g., Berton 2018; Paliya et al. 2018, and references therein). Their detection at radio and $\gamma$-ray bands has reinforced the view that in spite of being hosted by spiral galaxies, their central engines are capable of ejecting relativistic jets emitting strong nonthermal radiation, a hallmark characteristic of blazars (e.g., Yuan et al. 2008; Foschini et al. 2015). Their similarity to blazars is bolstered due to detection of the double-humped SED profile for several $\gamma$-rays NLSy1s (e.g., Abdo et al. 2009a,b; Foschini et al. 2011), in both flaring and non-flaring states (e.g., D'Ammando et al. 2016a; Paliya \& Stalin 2016). We note, however, that very recent studies have revealed that weak relativistic radio jets may even be launched by non-blazar type NLSy1 galaxies (Lähteenmäki et al. 2018).

Purely from the radio perspective, there is a clear evidence for (quasar-like) bi-modality in the radio loudness of NLSy1s. However, the radio-loud fraction is smaller; $\leqslant 7 \%$ NLSy1s have a radio-loudness parameter $\mathrm{R}_{5 \mathrm{GHz}}>$ 10 (e.g., Komossa et al. 2006; Zhou et al. 2006; Rakshit et al. 2017; Singh \& Chand 2018). This fraction is very similar to the radio-loud fraction of $4.7 \%$ (taking $\mathrm{R}_{5 \mathrm{GHz}}>10$ ) estimated by Rafter et al. (2009) for a flux-limited subset of 5477 broad-line AGN drawn from a low-z sample of AGNs, extracted by Greene \& Ho (2007) from the SDSS/DR4 (York et al. 2000; Adelman-McCarthy et al. 2006). In several radio-loud NLSy1s, kiloparsec-scale radio emission has been detected (e.g., Doi et al. 2012; Foschini et al. 2015; Congiu et al. 2017; Singh \& Chand 2018), although their flat spectrum subset exhibits very dim diffuse radio emission (Congiu et al. 2017; Berton 2018).

Even prior to the Fermi/LAT discovery of (variable) $\gamma$-ray emission, the flat/inverted radio spectra, high radio brightness temperatures, superluminally moving radio knots in the VLBI images of several NLSy1s, had become powerful indicators of relativistic jets in their cores (e.g., Zhou et al. 2003; Doi et al. 2006; Yuan et al. 2008; D'Ammando et al. 2013). Raising the radio-loudness threshold to $\mathrm{R}_{5 \mathrm{GHz}}>100$, which is probably a more secure criterion for radio loudness (e.g., Falcke et al. 1996b; Rafter et al. 2011), the radio-loud fraction of NLSy1s drops to just 2-3\% (Komossa et al. 2006). It is interesting to recall that Zhou et al. (2007) have argued that most of such 'very radio-loud' NLSy1s are in fact 'radio-intermediate' AGN

2 https://heasarc.gsfc.nasa.gov/docs/heasarc/missions/fermi.html 
with Doppler boosted nuclear radio emission. For quasars, the intermediate range of $R_{5 G H z}(10-100)$ has long been associated with 'radio intermediate' classification and they have even been postulated to be the tiny subset of normal QSOs whose intrinsically weak relativistic jets appear Doppler boosted as they happen to be pointed close to our direction (Miller et al. 1993; Falcke et al. 1996a; Wang et al. 2006). Irrespective of the underlying physical mechanism, it is a subset of clearly radio-loud and $\gamma$-ray NLSy1 galaxies, which will be the focus of the present study. Specifically, we shall present the results of our intranight optical monitoring (15 nights) of 3 such NLSy1 galaxies, having large $\mathrm{R}_{1.4 \mathrm{GHz}}$ (Table 1), all of which are also confirmed $\gamma$-ray emitters (Abdo et al. 2009c; Yao et al. 2015). In recent years it has often been pointed out for NLSy1s that their optical, near-infrared and even radio flux variability is similar to blazars (e.g., Liu et al. 2010; Jiang et al. 2012; Paliya et al. 2013; Angelakis et al. 2015). The 3 NLSy1s discussed in this paper constitute the radio loudest subset of the 25 NLSy1s which we are currently monitoring for intra-night and longer-term optical variability. They were extracted from the Foschini (2011) sample of 76 NLSy1s confirmed to be emitters of high-energy radiation: X-rays (detected with $\operatorname{ROSAT}^{3}$ ) and/or $\gamma$-rays (detected with FermiLAT). This paper is structured as follows. In Sect. 2 we describe our observations and the data reduction procedure, while Sect. 3 presents the statistical analysis of the light curves. Our main results and discussion are presented in Sect. 4, followed by conclusions in Sect. 5 .

\section{OBSERVATIONS AND DATA REDUCTION}

\subsection{Photometric Intranight Observations}

The 3 NLSy1 targets were monitored in the Johnson-Cousin $\mathrm{R}$ (hereafter $\mathrm{R}_{c}$ ) filter using the $1.3 \mathrm{~m}$ telescope (DFOT) of the Aryabhatta Research Institute of Observation Sciences (ARIES), India, located at Devasthal, Nainital (Sagar et al. 2010). DFOT is a fast beam (f/4) optical telescope with Ritchey-Chretien (RC) optics. It has a pointing accuracy better than 10 arcsec rms. The telescope is equipped with a $2 \mathrm{k} \times 2 \mathrm{k}$ deep thermo-electrically cooled (to about $-85^{\circ} \mathrm{C}$ ) Andor CCD camera with a pixel size of 13.5 microns and a plate scale of 0.53 arcsec per pixel, covering a FOV of $\sim 18 \mathrm{arcmin}^{2}$ on the sky. It has a readout speed of $1 \mathrm{MHz}$ and a system rms noise and gain of $7.5 e^{-}$and $2.0 e^{-} \mathrm{ADU}^{-1}$, respectively. We monitored each NLSy1 galaxy on 5 nights for $\geqslant 3.0$ hours, except for a slight shortfall in the duration, occurring in the case of the NLSy1 PKS J1222+0413 on 28.01.2017 due to poor weather conditions (Table 3). In order to get a reasonable SNR for each photometric measurement, the exposure times were typically set between 4 and 15 minutes, depending on the brightness of the source and the transparency and brightness of the sky.

\subsection{Data Reduction}

The pre-processing of the raw images (bias subtraction, flat-fielding and cosmic-ray removal) was done using the

\footnotetext{
3 https://heasarc.gsfc.nasa.gov/docs/rosat/rosat.html
}

standard tasks in the Image Reduction and Analysis Facility IRAF ${ }^{4}$. The instrumental magnitudes of the NLSy1 and stars in the image frames were determined by aperture photometry (Stetson 1987, 1992), using the Dominion Astronomical Observatory Photometry II (DAOPHOT II algorithm $)^{5}$. A crucial parameter for the photometry is the radius of the aperture which determines the $\mathrm{S} / \mathrm{N}$ ratio of the photometric data points for a given target. As suggested by Howell et al. (1988), the S/N ratio is maximised when the aperture radius approximately equals the full width at half maximum (FWHM) of the point-spread function (PSF) for the image (and decreases for both larger and smaller apertures). In order to find an optimum aperture, we have carried out aperture photometry, taking four aperture radii $=\mathrm{FWHM}, 2 \times \mathrm{FWHM}, 3 \times \mathrm{FWHM}$ and $4 \times$ FWHM. For each CCD frame, the value of FWHM (i.e., seeing disk radius) was determined by taking the mean over 5 fairly bright stars registered in the $\mathrm{CCD}$ frame. Although the photometric estimates using the different aperture radii were generally found to be in good agreement (see, also Sect. 4.1), the highest $\mathrm{S} / \mathrm{N}$ was almost always found when the aperture radius was set equal to $2 \times$ FWHM, which was hence adopted for the final analysis. As pointed out by Cellone et al. (2000), contamination from the host galaxy of the target AGN may result in spurious variability as the seeing disk varies, specially when the aperture is small. In the present sample, this issue is relevant in the case of the NLSy1 1H 0323+342 (z $=0.0629$ ) and has been specifically addressed in Sect. 4.2. In our analysis, we first found for a given session, the median of the FWHMs measured for all the CCD frames acquired in that session and used two times this median value as the aperture radius for the entire session. To derive the Differential Light Curves (DLCs) of a given target NLSy1, we selected two steady comparison stars (designated S1 and S2) present within all the CCD frames, such that they are close to the target NLSy1, both in location and apparent magnitude. We were able to ensure that at least one comparison star is within $\sim 1$ magnitude of the target NLSy1. The parameters of comparison stars selected for each session are given in Table 2. Note that the $g-r$ color difference for the target 'NLSy1' and the corresponding comparison stars is always $<0.80$ and $<1.80$ with the median values of 0.42 and 1.20 , respectively (column 7, Table 2). Analysis by Carini et al. (1992) and Stalin et al. (2004a), has demonstrated that color difference of this magnitude should produce a negligible effect on the DLCs, as the atmospheric attenuation changes during a monitoring session.

\section{STATISTICAL ANALYSIS OF THE DLCS}

Since the comparison stars are close in magnitude to the target NLSy1 in practically all the cases, as mentioned above (see, Table 2), the effects due to the difference in the photon noise are quite small. We shall, therefore, use the $F^{\eta}$-test (de Diego 2010) to check for the presence of INOV in the

\footnotetext{
4 Image Reduction and Analysis Facility (http://iraf.noao.edu/) ${ }^{5}$ Dominion Astrophysical Observatory Photometry (http://www.astro.wisc.edu/sirtf/daophot2.pdf)
} 
Table 1. Basic parameters of the three $\gamma$-ray NLSy1 galaxies and of their central engines.

\begin{tabular}{|c|c|c|c|c|c|c|c|c|c|c|}
\hline $\begin{array}{c}\text { Name (SDSS name) } \\
(1)\end{array}$ & $\begin{array}{l}\text { R.A.(J2000) } \\
(\mathrm{h} \mathrm{m} \mathrm{s}) \\
(2)\end{array}$ & $\begin{array}{c}\text { Dec.(J2000) } \\
\left(\begin{array}{c}0 \prime \prime \prime \\
(3)\end{array}\right. \\
(3)\end{array}$ & $\begin{array}{c}z \\
\text { (redshift) } \\
(4)\end{array}$ & $\begin{array}{c}\mathrm{m}_{B} \\
(\mathrm{mag}) \\
(5)\end{array}$ & $\begin{array}{c}\alpha_{\text {rad }}^{a} \\
(6)\end{array}$ & $\begin{array}{c}\mathrm{R}_{1.4 G H z}^{b} \\
(7)\end{array}$ & $\begin{array}{c}\mathrm{M}_{B H}^{c} \\
\left(10^{7} M_{\odot}\right) \\
(8)\end{array}$ & $\begin{array}{c}\lambda_{E d d}{ }^{d} \\
(9)\end{array}$ & $\begin{array}{c}{ }^{e} \Gamma_{S E D} \\
(10)\end{array}$ & $\begin{array}{c}\mathrm{PM}\left(\mathrm{yr}^{-1}\right)^{f} \\
(15 \mathrm{GHz}) \\
(11)\end{array}$ \\
\hline $1 \mathrm{H} 0323+342(\mathrm{~J} 032441.20+341045.0)$ & 032441.20 & +341045.00 & 0.063 & 16.38 & +0.1 & 318 & $(1-3)$ & 0.4 & $7-8^{*}$ & $9.0 c \pm 0.3 c$ \\
\hline PKS J1222+0413 (J122222.99+041315.9) & 122222.99 & +041315.95 & 0.966 & 17.88 & +0.3 & 1534 & 20 & 0.6 & 30 & $0.9 c \pm 0.3 c$ \\
\hline PKS J1505+0326 (J150506.48+032630.8) & 150506.48 & +032630.84 & 0.408 & 18.99 & +0.3 & 3364 & 4 & 0.1 & 17 & $1.1 \mathrm{c} \pm 0.4 \mathrm{c}$ \\
\hline
\end{tabular}

${ }^{a}$ Radio spectral index $\left(\mathrm{S}_{v} \propto v^{\alpha}\right)$ values for $1 \mathrm{H} 0323+342$, PKS J1222+0413 and PKS J1505+0326 are taken from

Neumann et al. (1994), White \& Becker (1992) and Angelakis et al. (2015), respectively.

${ }^{b} R_{1.4 \mathrm{GHz}}\left(f_{1.4 \mathrm{GHz}} / f_{4400 \AA}\right)$ values for $1 \mathrm{H} 0323+342$ and PKS J1505+0326 are taken from Foschini (2011) and

for PKS J1222+0413, $\mathrm{R}_{1.4 \mathrm{GHz}}$ value is estimated taking its core radio flux density of $0.6 \mathrm{Jy}$ at $1.4 \mathrm{GHz}$

(Kharb et al. 2010) and $\mathrm{f}_{v}(4400 \AA)$ from Yao et al. (2015).

${ }^{c}$ Black hole masses for 1H 0323+342, PKS J1222+0413 and are PKS J1505+0326 taken from Zhou et al. (2007),

Yao et al. (2015) and Paliya \& Stalin (2016), respectively.

${ }^{d}$ Eddington ratios for $1 \mathrm{H} 0323+342$, PKS J1222+0413 and PKS J1505+0326 are taken from Paliya et al. (2014),

Yao et al. (2015) and D'Ammando et al. (2016a), respectively.

${ }^{e}$ The bulk Lorentz factors $\left(\Gamma_{S E D}\right)$ for 1H 0323+342, PKS J1222+0413 and PKS J1505+0326 are taken from Paliya et al. (2014),

Yao et al. (2015) and D'Ammando et al. (2016a), respectively. The range (marked by *) encompasses the average and active states of $\gamma$-ray emission, with the higher value for the active state (Paliya et al. 2014).

${ }^{f}$ The VLBI radio knots proper motion measurements are from Lister et al. (2016). For 1H 0323+342, Fuhrmann et al. (2016),

reported its VLBI radio knots proper motions to be up to $\sim 7 \mathrm{c}$.

Table 2. Basic observational parameters of the comparison stars (S1, S2) used for the three $\gamma$-ray NLSy1 galaxies.

\begin{tabular}{|c|c|c|c|c|c|c|}
\hline $\begin{array}{l}\text { Name } \\
\text { (1) }\end{array}$ & $\begin{array}{l}\text { Dates of observations } \\
\qquad(2)\end{array}$ & $\begin{array}{l}\text { R.A.(J2000) } \\
\qquad(\mathrm{h} \mathrm{m} \mathrm{s}) \\
\quad(3)\end{array}$ & $\begin{array}{c}\text { Dec. }(\mathrm{J} 2000) \\
\left({ }^{\circ} \prime \prime \prime\right) \\
(4)\end{array}$ & $\begin{array}{c}\mathrm{g} \\
(\mathrm{mag}) \\
(5)\end{array}$ & $\begin{array}{c}\mathrm{r} \\
(\mathrm{mag}) \\
(6)\end{array}$ & $\begin{array}{r}\text { g-r } \\
(\mathrm{mag}) \\
(7)\end{array}$ \\
\hline $1 \mathrm{H} 0323+342$ & 22,23 Nov.; 02 Dec. 2016; 03, 04 Jan. 2017 & 032441.20 & +341045.00 & 14.50 & 13.70 & ${ }^{*} 0.80$ \\
\hline S1 & & 032453.68 & +341245.62 & 15.60 & 14.40 & ${ }^{*} 1.20$ \\
\hline S2 & & 032453.55 & +341116.58 & 16.20 & 14.40 & ${ }^{*} 1.80$ \\
\hline PKS J1222+0413 & 03, 04, 28 Jan.; 21, 22 Feb. 2017 & 122222.99 & +041315.95 & 17.02 & 16.80 & 0.22 \\
\hline S1 & & 122234.02 & +041321.57 & 18.63 & 17.19 & 1.44 \\
\hline S2 & & 122156.12 & +041515.19 & 17.22 & 16.78 & 0.44 \\
\hline PKS J1505+0326 & 25 March, 12, 21 April 2017; 11, 20 May 2018 & 150506.48 & +032630.84 & 18.64 & 18.22 & 0.42 \\
\hline S2 & & 150532.05 & +032836.13 & 18.13 & 17.64 & 0.49 \\
\hline S3 & & 150514.52 & +032456.17 & 17.51 & 17.14 & 0.37 \\
\hline
\end{tabular}

*Due to unavailability of SDSS (g-r) colors, (B-R) colors are taken from USNO-A2.0 catalog (Monet 1998).

DLCs, as discussed in Goyal et al. (2012). A specific advantage of this choice is that the results of our analysis for the NLSy1 galaxies can be readily compared with the INOV characterisation for other major classes of AGN, which has already been carried out in a uniform manner, employing the $F^{\eta}$-test (e.g., Goyal et al. 2013a). In this test, it is specially important to use the correct rms errors on the photometric data points. It has been found that the magnitude errors, returned by the routines in the data reduction software DAOPHOT and IRAF, are normally underestimated by a factor ranging between 1.3 and 1.75 (Gopal-Krishna et al. 1995; Garcia et al. 1999; Sagar et al. 2004; Stalin et al. 2004b; Bachev et al. 2005). Recently (Goyal et al. 2013b) have estimated the best-fit value of $\eta$ to be $1.54 \pm 0.05$, using 262 sessions of intranight monitoring of AGN.

The $F^{\eta}$-statistics can be written as (e.g., Goyal et al. 2012)
$F_{1}^{\eta}=\frac{\sigma_{(q-s 1)}^{2}}{\eta^{2}\left\langle\sigma_{q-s 1}^{2}\right\rangle}, \quad F_{2}^{\eta}=\frac{\sigma_{(q-s 2)}^{2}}{\eta^{2}\left\langle\sigma_{q-s 2}^{2}\right\rangle}, \quad F_{s 1-s 2}^{\eta}=\frac{\sigma_{(s 1-s 2)}^{2}}{\eta^{2}\left\langle\sigma_{s 1-s 2}^{2}\right\rangle}(1)$

where $\sigma_{(q-s 1)}^{2}, \sigma_{(q-s 2)}^{2}$ and $\sigma_{(s 1-s 2)}^{2}$ are the variances of the 'target-star1', 'target-star2' and 'star1-star2' DLCs and $\left\langle\sigma_{q-s 1}^{2}\right\rangle=\sum_{\mathbf{i}=0}^{N} \sigma_{i, e r r}^{2}(q-s 1) / N,\left\langle\sigma_{q-s 2}^{2}\right\rangle$ and $\left\langle\sigma_{s 1-s 2}^{2}\right\rangle$ are the mean square (formal) rms errors of the individual data points in the 'target-star1', 'target-star2' and 'star1-star2' DLCs, respectively. $\eta$ is the scaling factor and is taken to be 1.5 (see above).

The $F$-values are calculated for each DLC using Eq. 1 and compared with the critical $F$ value, $F_{v_{q s}, v_{s s}}^{(\alpha)}$, where $\alpha$ is the significance level set for the test, and $v_{q s}$ and $v_{s s}$ are the degrees of freedom for the 'target-star' and 'star-star' DLCs (both are equal in the present work). Here, we set two critical significance levels, $\alpha=0.01$ and 0.05 , which correspond to confidence levels of $99 \%$ and $95 \%$, respectively. Thus, we mark a NLSy1 as variable (V) if F-value is found to be $>$ 
Table 3. Observational details and the INOV results for the three $\gamma$-ray NLSy1s monitored in 15 sessions.

\begin{tabular}{|c|c|c|c|c|c|c|c|}
\hline $\begin{array}{c}\text { NLSy1 } \\
\text { (SDSS Name) } \\
(1)\end{array}$ & $\begin{array}{c}\text { Date } \\
\text { dd.mm.yyyy } \\
(2)\end{array}$ & $\begin{array}{l}\mathrm{T} \\
\mathrm{hr} \\
(3)\end{array}$ & $\begin{array}{c}\mathrm{N} \\
\text { Points in DLC } \\
(4)\end{array}$ & $\begin{array}{c}\mathrm{F}^{\eta} \text { values }^{a} \\
F_{1}^{\eta}, F_{2}^{\eta} \\
(5)\end{array}$ & $\begin{array}{l}\text { INOV status }^{b} \\
\mathrm{~F}^{\eta} \text {-test } \\
(6)\end{array}$ & $\begin{array}{c}\sqrt{\left\langle\sigma_{i, e r r}^{2}\right\rangle} \\
(\mathrm{q}-\mathrm{s}) \\
(7)\end{array}$ & $\begin{array}{c}\bar{\psi}_{s 1, s 2} \\
(\%) \\
(8)\end{array}$ \\
\hline \multirow[t]{5}{*}{$1 \mathrm{H} 0323+342$} & 22.11 .2016 & 4.42 & 56 & $1.68,2.80$ & $P V, V$ & 0.007 & 4.5 \\
\hline & 23.11.2016 & 4.27 & 54 & $3.18,4.31$ & $\mathrm{~V}, \mathrm{~V}$ & 0.006 & 4.7 \\
\hline & 02.12 .2016 & 4.41 & 44 & $11.00,11.50$ & $\mathrm{~V}, \mathrm{~V}$ & 0.008 & 9.34 \\
\hline & 03.01.2017 & 3.00 & 39 & $1.03,1.16$ & $\mathrm{NV}, \mathrm{NV}$ & 0.009 & - \\
\hline & 04.01.2017 & 3.39 & 33 & $3.50,3.47$ & $\mathrm{~V}, \mathrm{~V}$ & 0.009 & 6.8 \\
\hline \multirow[t]{5}{*}{ PKS J1222+0413 } & 03.01.2017 & 3.52 & 17 & $0.53,0.25$ & $\mathrm{NV}, \mathrm{NV}$ & 0.020 & - \\
\hline & 04.01.2017 & 3.62 & 17 & $0.31,0.36$ & NV, NV & 0.014 & - \\
\hline & 28.01.2017 & 2.45 & 23 & $0.56,0.52$ & $\mathrm{NV}, \mathrm{NV}$ & 0.022 & - \\
\hline & 21.02.2017 & 4.44 & 41 & $0.74,0.76$ & $\mathrm{NV}, \mathrm{NV}$ & 0.020 & - \\
\hline & 22.02.2017 & 5.50 & 50 & $3.68,3.50$ & $\mathrm{~V}, \mathrm{~V}$ & 0.018 & 13.2 \\
\hline \multirow[t]{5}{*}{ PKS J1505+0326 } & 25.03.2017 & 5.34 & 42 & $0.62,0.63$ & $\mathrm{NV}, \mathrm{NV}$ & 0.029 & - \\
\hline & 12.04 .2018 & 3.97 & 22 & $0.38,0.51$ & $\mathrm{NV}, \mathrm{NV}$ & 0.040 & - \\
\hline & 21.04.2018 & 5.18 & 25 & $0.50,0.50$ & $\mathrm{NV}, \mathrm{NV}$ & 0.038 & - \\
\hline & 11.05 .2018 & 3.16 & 14 & $1.80,1.45$ & $\mathrm{NV}, \mathrm{NV}$ & 0.037 & - \\
\hline & 20.05.2018 & 3.30 & 15 & $0.60,0.37$ & $\mathrm{NV}, \mathrm{NV}$ & 0.045 & - \\
\hline
\end{tabular}

$F_{c}(0.99)$ for both its DLCs (relative to the two comparison stars), non-variable (NV) if any one out of two DLCs is found to have F-value $<F_{c}(0.95)$. The remaining cases are designated as probably variable (PV). The computed $F$-values and the corresponding INOV status for the $3 \gamma$-ray NLSy1s are given in columns 5 and 6 of Table 3 .

For computing the amplitude $(\psi)$ of INOV we have followed the definition given by Heidt \& Wagner (1996)

$$
\psi=\sqrt{\left(D_{\max }-D_{\min }\right)^{2}-2 \sigma^{2}}
$$

with $D_{\min , \max }=$ minimum (maximum) values in the NLSy1star DLC and $\sigma^{2}=\eta^{2}\left\langle\sigma_{q-s}^{2}\right\rangle$, where, $\left\langle\sigma_{q-s}^{2}\right\rangle$ is the mean square (formal) rms error of individual data point and $\eta$ $=1.5$ (Goyal et al. 2013b).

\section{RESULTS AND DISCUSSION}

It is worth reiterating that even though $\gamma$-ray loud NLSy1s display blazar-like properties, their studies carry a special interest because their AGNs reside in spiral galaxies (Sect. 1) and thus the jets are launched into a much denser environment than is the case for blazars whose hosts are nearly always early-type galaxies (see Sect. 1; also, Bagchi et al. 2014, and references therein). The use of INOV as a tracer of blazarlike jet activity in $\gamma$-ray NLSy1s became popular around the beginning of this decade. For the NLSy1 PMN J0948+0022, Liu et al. (2010) observed a brightness change of $\sim 0.5$ mag over several hours (also, Eggen et al. 2013; Paliya et al. 2013; Liu et al. 2016). Similarly, violent INOV events have since been reported for a few other $\gamma$-ray NLSy1s, such as $1 \mathrm{H}$ 0323+342 (Paliya et al. 2014) and SBS 0846+513 (hereafter J0849+5108) (e.g., Maune et al. 2014; Paliya et al. 2016). It is now known that the duty cycle (DC) of INOV for radioloud NLSy1s is around $\sim 50 \%$, with somewhat higher values found for their $\gamma$-ray detected subset (Paliya et al. 2013; Kshama et al. 2017). This, too, mirrors the situation known for blazars (e.g., Stalin et al. 2005; Lister et al. 2009; Pushkarev et al. 2009). Below we summarize some salient aspects of the $3 \gamma$-ray NLSy1s as well as their variability properties found in the present study.

\subsection{The NLSy1 1H $0323+342(z=0.0629)$}

Multiple mass estimates for the central BH of this NLSy1 galaxy fall in the range $(1-3) \times 10^{7} M_{\odot}$ (Zhou et al. 2007) which, although normal for NLSy1s (Sect. 1), is on the lower side for blazars (Sect. 1). Correspondingly, it is operating at a high Eddington ratio of $\lambda_{E d d} \sim 0.4$ (Paliya et al. 2014), which again is not exceptional for NLSy1s (Boroson 2002; Grupe \& Mathur 2004). A blazar description of this NLSy1, other than its $\gamma$-ray flaring (Carpenter \& Ojha 2013; Paliya et al. 2014), stems from the flatness of its radio spectrum up to $10 \mathrm{GHz}\left(\alpha_{r}=+0.1\right.$, Neumann et al. 1994) and even higher radio frequencies (Angelakis et al. 2015). Another evidence for a relativistically beamed jet comes from its VLBI image, which shows a radio core with one-sided jet (e.g., Lister \& Homan 2005; Zhou et al. 2007). In their detailed VLBI study of this source at $15 \mathrm{GHz}$, Fuhrmann et al. (2016) have resolved the jet into 7 well-aligned knots and estimated them to have proper motions of up to 7c. They also estimate the jets viewing angle to be within $\sim 13$ degrees. Furthermore, in the states of both average and high $\gamma$-ray activity, its dual-humped SED showed the synchrotron component peaking near $10^{12.5} \mathrm{~Hz}$, which is a characteristic of FSRQ/LBL type AGN (Zhou et al. 2007; Paliya et al. 2014).

By comparing the SED for its nucleus with other AGN, Zhou et al. (2007) concluded that its optical light is dominated by thermal emission, as also independently inferred by Paliya et al. (2014) from their SED modeling for 
both the average and high-activity states. The contamination from this thermal emission of Seyfert origin is probably responsible for the observed low optical polarization $<1 \%$ (Eggen et al. 2011; Ikejiri et al. 2011). Even during the high activity state detected by Fermi/LAT in July 2013, which lasted $\sim 20$ days, its polarization rose only to $\sim 3 \%$ (Itoh et al. 2014). A similarly low polarisation has been reported by Pavlidou et al. (2014) and, more recently by Angelakis et al. (2018). At radio wavelengths too, the source exhibited a rather modest polarisation $\left(\mathrm{p}_{\text {rad }} \sim\right.$ $4 \%$ at $10.55 \mathrm{GHz}$, Neumann et al. 1994). Recall, however, that polarisation dips are not unexpected for NLSy1 galaxies (Eggen et al. 2011; Ikejiri et al. 2011; Itoh et al. 2013, 2014). Even for blazars, Fugmann (1988) has shown that there is $40 \%$ chance that a bona fide blazar will appear only weakly polarised ( $\mathrm{p}_{\text {opt }}<3 \%$ ) at a random epoch. Recall also that even the very prominent BL Lac object OJ 287 has sometimes been found to be unpolarized (e.g., Villforth et al. 2009).

Taking only the unbeamed radio flux, Zhou et al. (2007) found an $R_{5 G H z}$ between 4 and 25, placing this NLSy1 in the category of 'radio intermediate quasars' (RIQs) (Miller et al. 1993; Falcke et al. 1996a,b; Diamond-Stanic et al. 2009). Here we may note the extensive dataset on intranight optical monitoring of RIQs, published by Goyal et al. (2010), has demonstrated that their INOV generally maintains a low level, both in amplitude $(\psi<3 \%)$ and duty cycle (DC $\sim 10 \%)$. Putting together this and the modest polarisation, it would seem unlikely that a strong INOV activity can be witnessed in this NLSy1. However, as we discuss below, this somewhat discouraging prognosis was overturned by the two episodes of violent optical variability recorded for this NLSy1 in separate intranight monitoring sessions $\sim 4$ years apart.

The first INOV study of this $\gamma$-ray NLSy1 was reported by Paliya et al. (2013) who monitored it on 4 nights within a span of 10 days during early 2012. The observations on two of the nights were quite noisy and in the remaining two nights, the monitoring duration exceeded our threshold of 3 hours just on the night of 26.01.2012, when the source showed a strong INOV $(\psi \sim 7 \%)$. In another campaign during late 2012, the source was monitored by them on 3 nights (Paliya et al. 2014). On the first two nights, only mild INOV was observed, however, on the third night (09.12.2012) the DLCs taken with a temporal resolution of 2 minutes, showed a very strong outburst, when the optical flux rose by $\psi \sim 35 \%$ within just $\sim 30$ minutes. The import of this and a similar INOV event detected in the present study of this NLSy1 is discussed below.

In our campaign during 2016-17, we monitored this NLSy1 on 5 nights, each time for $\geqslant 3$ hours (Table 3). Strong INOV, with $\psi$ between $4 \%$ and $9 \%$ was detected on 4 of the 5 nights (Table 3; Fig. 1 \& 2, see below), which is clearly reminiscent of blazars (e.g., Goyal et al. 2013a). In all these sessions, seeing disc variations were quite small; the seeing data for the night of 02.12.2016 are plotted in Fig. 3. It may be recalled that the issue of variable seeing is specially relevant for nearby AGN like this NLSy1, for which the host galaxy can be a significant contributor to the aperture photometric measurements (Cellone et al. 2000). Fortunately, an HST image is available for this NLSy1, and it shows that the host galaxy, with a total extent of 15 arcsec, contributes close to $50 \%$ of the optical flux, the remainder coming from the AGN (Zhou et al. 2007). As discussed below, this informa- tion plays a key role in a quantitative interpretation of the INOV and other observations of this AGN.

To further check the possible impact of seeing variations on our DLCs, we have derived a new set of DLCs taking larger photometric apertures (i.e., radius $=4,6$ and 7 times the median FWHM found for the given session). These DLCs confirm the strong INOV seen in the DLCs of this source (see Fig. 1), for all the nights, excepting the night of 03.01.2017 (Table 3). Focussing next on the session on 02.12.2016 when a large optical outburst was seen, Fig. 3 shows that the base level of the 'AGN - star' DLCs stops rising further significantly when the aperture radius crosses $6 \times$ FWHM (15.6 arc$\mathrm{sec}$ ). This means that this aperture is large enough to pick virtually the entire emission from the host galaxy (which is consistent with its size in the HST image taken by Zhou et al. (2007).

To summarize, the present INOV observations have confirmed that this NLSy1 galaxy is capable of strong INOV activity, with a high DC $\sim 60$ to 75 per cent. Earlier, a similarly intense INOV activity has been observed for the proto-typical NLSy1s J0849+5108 (Maune et al. 2014). Such large and frequent INOV is strikingly reminiscent of some prominent BL Lacs, like AO 0235+164 and OJ 287 (e.g., Romero et al. 2000; Sagar et al. 2004; Goyal et al. 2017; Britzen et al. 2018).

\subsection{The spectacular optical outburst of $1 \mathrm{H} 0323+342$}

Although INOV was detected for the NLSy1 galaxy $1 \mathrm{H}$ $0323+342$ on 4 of the 5 nights we monitored it, the most spectacular variation occurred on 02.12.2016. During the 4.4 hours of continuous monitoring with high-sensitivity, both comparison stars remained rock steady and the seeing disk, too, was steady (Fig. 3). Nearly in the middle of the session, a large, roughly flat-topped and nearly symmetric outburst of total duration $\sim 1.25$ hours was observed. For the DLCs corresponding to the aperture radius of $6 \times$ FWHM (see above), the rising phase of the outburst, which is temporally resolved, shows a 0.07 mag variation occurring within at most 20 minutes and a similarly steep gradient was seen for the declining phase which, too, is resolved temporally. Such sharp variations are extremely rare episodes even for blazars (e.g., Gopal-Krishna \& Wiita 2018, and references therein). Curiously, this outburst bears an uncanny resemblance to the one this NLSy1 had exhibited on 09.12.2012 (Fig. 10 of Paliya et al. 2014) coinciding with a $\gamma$-ray flare (note that the precursor optical bump seen in the DLCs on that night is most probably an artefact due to the sudden spell of seeing disk deterioration, which can be seen in the bottom panel of their Fig. 10). During that outburst, this NLSy1 brightened by $\sim 0.35 \mathrm{mag}$ in 30 minutes and after remaining at the elevated brightness for $\sim 1.1$ hours, reverted almost as rapidly to its initial level. While the amplitudes of these two optical outbursts are impressive, they are by no means exceptional for $\gamma$-ray NLSy1s. For instance, two INOV flares of $\psi \sim 0.3 \mathrm{mag}$, with a rise/fall time between 10-30 minutes were detected during the intranight monitoring of the NLSy1 PMN J0948+0022 on 01.04.2011 (Eggen et al. 2013). Earlier, Liu et al. (2010) had reported for the same NLSy1 a brightness change of $\sim 0.5$ mag over several hours. Likewise, during a high $\gamma$-ray activity phase, the NLSy1 J0849+5108 was found to fade by $\sim 0.2$ mag within just $\sim 15 \mathrm{~min}-$ 
utes (figure 6 of Maune et al. 2014). As we shall now argue for $1 \mathrm{H} 0323+342$, the 0.07 mag brightening within at most 20 minutes, seen at the beginning of the 02.12 .2016 outburst (Fig. 2 \& 3), actually corresponds to a remarkably short flux doubling time of $\sim 1$ hour for this AGN's nonthermal output. This conclusion is reached when we subtract out from the aperture photometric measurements, the expected contributions of thermal optical emission, made by the host galaxy and by the accretion disk associated with the Seyfert nucleus (even which is not expected to vary by $>1 \%$ on hourlike time scale; see Mangalam \& Wiita 1993). To get an idea of the accretion disc's contribution, we return to the modeling of this AGN's SEDs for the four epochs, which revealed that in both high and low states of $\gamma$-ray activity, the optical emission was dominated by the thermal component contributed by its Seyfert nucleus (Paliya et al. 2014, their Fig. 9), in agreement with the conclusion reached earlier by Zhou et al. (2007). Accordingly, we shall make a conservative assumption that $50 \%$ of the optical emission from the AGN is thermal and only the remainder is synchrotron light. Next, consider the HST image of this NLSy1 which showed that the total optical emission from the (unresolved) AGN is essentially equal to that arising from the underlying host galaxy $\sim 15$ arcsec in diameter (Zhou et al. 2007). As discussed above, essentially all this emission from the host galaxy has got picked up in our photometry with a circular aperture of radius $6 \times \mathrm{FWHM} \sim 15.6$ arcsec. Thus, putting together the likely thermal contributions to the DLCs of this NLSy1 (Fig. 3), which come from the Seyfert nucleus and the host galaxy, it can be concluded that only $\sim 25 \%$ or less of the amplitude of the light curves is of synchrotron origin and the observed rapid outburst and other observed INOV is associated entirely with this minor component. Therefore, in order to account for the observed brightening by $\sim 7 \%$ at the beginning of the optical outburst, the optical synchrotron component of the AGN is required to have brightened up (in $<20$ minutes, see above) by a factor of 1.27 . This corresponds to a flux doubling time of $\sim 1$ hour. Such INOV can only be described as extreme, as it is about 20 times larger in amplitude than that typically displayed by blazars (e.g., Ferrara et al. 2001; Goyal et al. 2012). If, in a less conservative vain, we consider the share of synchrotron optical emission in the total optical output of the AGN to be less than 50\% (see, e.g., Paliya et al. 2014), the deduced flux doubling time would be shorter still.

Note that a very similar conclusion can be drawn from the optical flare exhibited by this NLSy1 on 9.12.2012 (Fig. 10 of Paliya et al. 2014). During that flare, the source brightened up by $\sim 35 \%$ in just 30 minutes. As opposed to our DLCs displayed in Fig. 2 \& 3, those DLCs are based on photometry with a much smaller aperture; indeed, they are claimed by Paliya et al. (2014) to essentially represent just the AGN component of the emission, which they assert is dominated by thermal radiation. Before proceeding further, we make two conservative assumptions about those DLCs of J0324+3410, namely that (a) they have zero contribution from the host galaxy and (b) that $50 \%$ (i.e., the maximum permissible) of the amplitude of each DLC is due to the AGN's synchrotron radiation. Even on this grossly conservative basis, it is evident that, in order to cause the observed brightness change of $\sim 35 \%$ in 30 minutes, the AGN's synchrotron optical emission must have increased by at least $\sim 70 \%$ (within the 30 minutes). This corresponds to a flux doubling time of $\sim 0.7$ hours, a yet another exceptional event exemplifying once again the extreme INOV behaviour of this NLSy1. In reality, the flux doubling times for both this flare and the one reported here (Fig. $2 \& 3$ ) may be substantially shorter than the present conservative estimates of $\leqslant 1$ hour, thereby approaching the extremely fast variability of $\mathrm{GeV} / \mathrm{TeV}$ radiation detected for some blazars (e.g., a flux doubling time of $\sim$ 10 minutes observed for the blazar PKS $1222+21$ at $400 \mathrm{GeV}$, by Aleksić et al. 2011; Ackermann et al. 2014).

\subsection{The NLSy1 PKS J1222 $+0413(z=0.966)$}

This is the farthest known $\gamma$-ray-emitting NLSy1. Aside from its $30 \sigma$ level of $\gamma$-ray detection (Ackermann et al. 2015; Yao et al. 2015, hereafter YOF15) and the VLBI detection of a one-sided jet (Lister et al. 2016), evidence for relativistic jet in this NLSy1 comes also from its extreme radio loudness $\left(R_{5 G H z} \sim 1700\right.$ for the core, YOF15), a relatively flat hard X-ray spectrum (photon index $\Gamma \sim 1.3$, YOF15) which is typical of inverse Compton X-rays from relativistic jets, and an inverted radio spectrum $\left(\alpha_{r}=+0.3\right.$ between 1.4 and $4.9 \mathrm{GHz}$, White \& Becker 1992). Others evidence include a high brightness temperature of its VLBI core $\left(\sim 4 \times 10^{12} \mathrm{~K}\right.$ at $8.6 \mathrm{GHz}$, Pushkarev \& Kovalev 2012) and a rather large long-term radio flux variability, ranging from $\sim 0.5$ to 1.1 Jy at $5 \mathrm{GHz}$ (YOF15). The synchrotron bump in its bi-modal SED peaks in the infrared (YOF15), a hallmark of LBL type blazars (Urry \& Padovani 1995; Abdo et al. 2010) which are known to exhibit the strongest optical variability among all AGN classes, both on hour-like and longer time scales (e.g., Heidt \& Wagner 1996; Hovatta et al. 2014).

Modeling the SED in terms of one-zone leptonic relativistic jet undergoing external Compton losses has yielded a bulk Lorenz factor $\Gamma \sim 30 \pm 5$ (YOF15) which is very close to the similarly estimated $\Gamma \sim 30$ for the well-known radioloud $\gamma$-ray NLSy1 PMN J0948+0022 (D'Ammando et al. 2015a), but much larger than $\Gamma<15$ which is typical for $\gamma$-ray NLSy1s (e.g., Abdo et al. 2009b; D'Ammando et al. 2012). Interestingly, a similar excess exists even in the $\mathrm{BH}$ mass. The best virial estimates, based on optical spectra, are (1-2) $\times 10^{8} M_{\odot}$ for PKS J1222+0413 (YOF15, Sbarrato et al. 2012) and $\sim 10^{8} M_{\odot}$ for the archetypal $\gamma$-ray NLSy1 PMN J0948+0022 (Zhou et al. 2003). These are much higher than the typical value $\left(\mathrm{M}_{B H} \sim 10^{7} \mathrm{M}_{\odot}\right)$ reported for NLSy1s (e.g., Yuan et al. 2008) although these estimates could be systematically low for reasons mentioned in Sect. 1 (e.g., see Calderone et al. 2013), albeit contested by others (e.g., Jarvela et al. 2017). In spite of the atypically high $\mathrm{M}_{B H}$, the central engine of PKS J1222+0413 is found to operate at a very high Eddington ratio ( $\left.\lambda_{E d d} \sim 0.6, \mathrm{YOF} 15\right)$.

To our knowledge, the present study is the first characterisation of the INOV of this NLSy1. Out of our 5 nights of monitoring, during January-March (2017), INOV was seen in just one session (22.02.2017) when the source showed a steady brightening by $\sim 0.1$ mag over 3 hours (Fig. 1). Excluding this session with a clear INOV detection, the maximum brightness change witnessed across the remaining 4 sessions is only $\sim 0.08 \mathrm{mag}$, which is not excessive even for radio-quiet quasars of low-luminosity/redshift (e.g., Caplar et al. 2017). Note, however, that the 3.4 and $4.6 \mathrm{mi}-$ 
cron data in the WISE catalogue (Wright et al. 2010) have shown an intra-day variation with $\psi \sim 50 \%$ (YOF15), which is clearly blazar-like and a similar large amplitude has been witnessed in the radio band where its $5 \mathrm{GHz}$ emission was found to vary in the range from $\sim 0.5$ to 1.1 Jy over a time scale of years (YOF15).

\subsection{The NLSy1 PKS J1505+0326 $(z=0.4089)$}

The Fermi/LAT detection of this 'extremely radio-loud' $\left(\mathrm{R}_{1.4 \mathrm{GHz}} \sim\right.$ 3364) NLSy1 (Abdo et al. 2009c) constitutes a strong evidence for a relativistically boosted nonthermal jet. Additional evidences include the relatively hard X-ray spectrum (D'Ammando et al. 2013), an inverted radio spectrum up to $\sim 10 \mathrm{GHz}\left(\alpha_{r} \sim+0.3\right)$ and a high brightness temperature inferred from flux variability at 2.6 $\mathrm{GHz}, \sim 2.6 \times 10^{13} \mathrm{~K}$ (Angelakis et al. 2015). Another indication of a high Doppler factor (between 3.9 and 6.6) has come from radio flux variability at $15 \mathrm{GHz}$ during the $\gamma$-ray outbursts (D'Ammando et al. 2013, 2016a, hereafter DOF16), invoking the concept of 'equipartition Doppler Factor' (Singal \& Gopal-Krishna 1985; Readhead et al. 1996). From VLBI, the source is also known to exhibit a bright core plus a weak jet at $15 \mathrm{GHz}$ (D'Ammando et al. 2013; Orienti et al. 2012). However, the VLBI images collected during 2010 - 2013 under the MOJAVE ${ }^{6}$ programme (Lister et al. 2016) have revealed only a sub-luminal component in its radio jet, with a proper motion of $1.1 \mathrm{c} \pm 0.4 \mathrm{c}$. The only major $\gamma$ ray flaring activity of this NLSy1, recorded by Fermi/LAT, occurred during Dec. 2015 to Jan. 2016 (D'Ammando \& Ciprini 2015) when its light-curve plotted in 3-hour bins showed a brightening by almost 2 orders of magnitude, although no significant signal was detected on shorter timescale. Multiband observations triggered by this rare flaring event have been reported by Paliya \& Stalin (2016) and DOF16. The SEDs during the flare activity and the state of average activity, both show the synchrotron bump peaking near $10^{13.5}$ $\mathrm{Hz}$, which is characteristic of FSRQs / LBLs and unlike HBLs. This conclusion is reinforced by its observed similarity to LBLs in terms of $\gamma$-ray luminosity, photon index and Compton dominance (Ackermann et al. 2015; Paliya \& Stalin 2016; D'Ammando et al. 2016a).

From modeling the SED's optical/UV bump as ShakuraSunyaev accretion disk, $M_{B H} \sim 4 \times 10^{7} M_{\odot}$ has been estimated by Paliya \& Stalin (2016), which is $\sim 2$ times the virial estimate reported by Shaw et al. (2012) based on the $\mathrm{Mg}$ II line. Taking this $\mathrm{M}_{B H}$ and modeling the SED in terms of the standard single leptonic blob moving in a relativistic jet close to our direction and producing synchrotron selfCompton (SSC) and external Compton (EC) emissions (e.g., Tavecchio et al. 2001; Finke et al. 2008; Dermer et al. 2009; Abdo et al. 2011), yields $\Gamma_{\text {jet }} \sim 17$ during the high activity state, which corresponds to an Eddington ratio of $\sim 0.1$ (DOF16).

In the optical band, this NLSy1 has been under regular monitoring since 2008 in the CRTS (Drake et al. 2009). Until 2016 a total variability amplitude of 0.7 mag has thus been recorded (DOF16), which is blazar-like (see, e.g.,

${ }^{6}$ Monitoring Of Jets in Active galactic nuclei with VLBA Experiments (https:/ / www.physics.purdue.edu/MOJAVE/)
Kügler et al. 2014). On the intranight scale, prior to our observations, Paliya et al. (2013) had monitored this NLSy1 on 4 nights during April-May (2012) which coincided with its moderately active $\gamma$-ray phase (DOF16). While the resulting optical DLCs are not available, making it hard to assess their sensitivity level and cadence, the authors have reported a clear INOV detection with $\psi \sim 10 \%$ on one of the 4 nights (24.05.2012). This amplitude too, is blazarlike (see, Ruan et al. 2012; Goyal et al. 2013a; Kügler et al. 2014). Paliya et al. (2013) also detected an episode of (mild) inter-day optical variability of $\sim 0.045 \mathrm{mag}$ and this too is on the higher side for radio-quiet quasars, which are known to vary typically by atmost $1-2 \%$ on 1 -day time scale (e.g., Caplar et al. 2017). The results of our monitoring of this extremely radio-loud NLSy1 on 5 nights are displayed in Fig. 1. The only significant variability is the $\sim 0.1$ mag brightness change between 21.04.2018 and 11.05.2018, representing short-term optical variability (STOV) on day-like time scale.

In summary, it seems fair to conclude that during our 5 nights' optical monitoring, the several previously known blazar-like attributes of this extremely radio-loud NLSy1 (Sect. 4.1) were not robustly reflected in its INOV and longerterm optical variability, which stands in stark contrast to the NLSy1 1H 0323+342, the least radio-loud member of the present set of $3 \gamma$-ray NLSy1s. As a caveat, we would like to mention that the much lower level of INOV shown by PKS $\mathrm{J} 1505+0326$ may have to be revised upwards if in future it becomes possible to reliably account for the dilution by the thermal optical output of its AGN, for which a hint already exists (D'Ammando et al. 2016b).

\subsection{Comments on the INOV diversity of the $3 \gamma$-ray NLSy1s}

The 3 Fermi/LAT detected NLSy1 galaxies (Table 1) studied here, share a number of observational commonalities, like highly significant $\gamma$-ray and radio detection, a flat/inverted radio spectrum (which flags dominance by a parsec-scale, or more compact radio jet). Additionally, their broadband SEDs are not only blazar-like (with a dual-hump profile) but also the peaking of the synchrotron hump in the infrared in each case, means that all 3 NLSy1s are counterparts of the FSRQ/LBL subclass of blazars and hence expected to exhibit strong radio/optical flux variability (e.g., Heidt et al. 1997; Maune et al. 2014). In the present study, a blazar-like intense and frequent INOV (duty cycle $\sim 60-75 \%$ ) was detected only for the NLSy1 $1 \mathrm{H} 0323+342$. Remarkably, not only is this NLSy1 the least radio-loud among the 3 NLSy1s reported here, a recent study has even re-classified it as a 'radio intermediate' with $\mathrm{R}_{5 \mathrm{GHz}}$ between 4 and 25 , based on its unboosted radio emission (Zhou et al. 2007). Even starker manifestations of its violent INOV are encapsulated in the two optical outbursts for which the present analysis conservatively implies a doubling time of $\sim 1$ hour for the optical synchrotron flux (Sect. 4.2). Such an INOV activity is intriguing for a 'radio intermediate' since an extensive study has shown that, in general, radio-intermediate quasars exhibit only lowlevel INOV $(\psi<3 \%)$ and the duty cycle is also small $(\sim 10 \%$, see Goyal et al. 2010). The contrary behaviour of the NLSy1 J0324+3410 indicates that a large radio loudness parameter per se is not an essential pre-requisite for strong INOV in the case of NLSy1 galaxies. Even for radio-loud quasars, a high 
radio loudness does not guarantee a strong INOV, as emphasized in Goyal et al. (2012), (see also, Gopal-Krishna \& Wiita 2018). Specially in the context of NLSy1 galaxies, a closer look into the dependence of INOV on radio-loudness would need a more realistic estimation of radio-loudness, since the required exclusion of the Doppler boosted anisotropic radio emission can make a big difference, given the low prominence of diffuse radio emission in NLSy1s with prominent jets (Doi et al. 2012; Congiu et al. 2017).

For $\gamma$-ray NLSy1s, uncertainty in $\mathrm{R}_{5 \mathrm{GHz}}$ is not the only complicating factor in probing the dependence of INOV on radio loudness. As highlighted above from the example of $1 \mathrm{H} 0323+342$, the observed INOV amplitude may itself be significantly, if not grossly, underestimated, depending on the extent to which the AGN's rapidly varying synchrotron optical luminosity (arising from its relativistic jet) gets contamination by the AGN's thermal light. Even for the radio detected NLSy1s, there is growing evidence for a significant, if not dominant thermal component in the AGN's optical output (e.g., D'Ammando et al. 2012; Sbarrato et al. 2018), even in an active state (e.g., Zhou et al. 2007; Paliya et al. 2014). Another potentially serious observational repercussion of such a thermal contamination is the dilution of the AGN's optical polarization signal, as emphasized in Sect. 4.1 for the specific case of the NLSy1 $1 \mathrm{H} 0323+342$. Such a camouflaging of the blazar lurking inside the nucleus is also known to occur in some quasars (e.g., Giroletti \& Panessa 2009; Antonucci 2012), 3C273 being a prime example (Impey et al. 1989; Valtaoja et al. 1990; Courvoisier 1998). But, this problem is probably more prevalent in the case of radio-loud NLSy1s, since they are generally high Eddington-rate accretors (e.g., Foschini et al. 2015), hence expected to generate a larger thermal radiation in the Seyfert nucleus (besides intermittent jet activity, see, Czerny et al. 2009). Thus, at least for defining the polarisation status, radio band may offer a more fruitful channel, since any thermal contamination by the AGN is expected to be far less substantial, although perhaps not vanishingly small (see, e.g., Laor \& Behar 2008). Another challenge is to arrive at a proper estimate of radio-loudness (i.e., $\mathrm{R}_{5 \mathrm{GHz}}$ ) for which knowledge of the unbeamed radio emission is vital. Mostly, this would require sensitive VLBI observations, given that the radio spectrum associated with $\gamma$ ray NLSy1s is usually flat or inverted (e.g., Angelakis et al. 2015), which means that the unbeamed radio emission is either faint, or compact (or, both). All this underscores the increasing role of radio observations in probing the physics of NLSy1s.

Nevertheless, one point that stands out from Table 1, is that the NLSy1 $1 \mathrm{H} 0323+342$, the solitary AGN showing a large and frequent INOV in our program thus far, is also the only one for which VLBI monitoring has revealed a large apparent superluminal motion in the jet ( $\mathrm{v} \sim 9 \mathrm{c})$; the other two NLSy1s (PKS J1222+0413 and PKS J1505+0326) have only displayed a sub-luminal synchrotron jet (of radio/optical emission, see Table 1). Here, it needs to be clarified that although the bulk Lorenz factors for these two sources, as determined from SED modeling are actually very large $(\Gamma \sim 30$ and $\sim 17)$, these values are more representative of the parts of the jet which produce high-energy photons and probably not closely linked to the radio/optical emitting zones of the relativis- tic jet (e.g., Ghisellini et al. 2005). It is also interesting to consider the other two well known Fermi/LAT NLSy1s, namely J0849+5108 and PMN J0948+0022, both of which have exhibited intense INOV (e.g., Eggen et al. 2013; Maune et al. 2014). Their VLBI monitoring at $15 \mathrm{GHz}$ under the MOJAVE programme has demonstrated that both are superluminal, with an apparent speed of $5.8 \mathrm{c} \pm 0.9 \mathrm{c}$ for J0849+5108, and $11.5 c \pm 1.5 c$ for PMN J0948+0022 (Lister et al. 2016). Thus, at present, no example of $\gamma$-ray emitting NLSy1s is known where a strong INOV is associated with a radio jet lacking apparent superluminal motion. It was noted above that the persistent violent INOV activity of $1 \mathrm{H} 0323+342$ is mirrored in the $\mathrm{BL} \mathrm{Lac} \mathrm{object} \mathrm{AO} 0235+164$. It is interesting that this blazar has displayed an ultra-fast apparent superluminal speed of $\sim 46$ c (Jorstad et al. 2001).

In summary, the present study of 3 Fermi/LAT NLSy1 galaxies is suggestive of a physical link between violent INOV and apparent superluminal motion, two key observables for active galactic nuclei. This is not to contend that the INOV relates weakly to other important parameters, e.g., optical polarization, which would be in clear conflict with the strong correlation found in the case of radio-loud quasars (e.g., Goyal et al. 2012). But, as mentioned above, verifying such a linkage in the case of NLSy1s would require a more concerted radio/optical follow-up that would enable a fairly precise separation of the synchrotron and thermal radiations in the SED of the Seyfert nucleus. The challenges in realising this make NLSy1 galaxies important targets for multi-wavelength, time-domain astronomy.

\section{CONCLUSIONS}

In summary, the present 15 sessions of intranight optical monitoring of $3 \gamma$-ray detected radio-loud NLSy1 galaxies have raised a couple of somewhat unexpected but significant issues. One of them relates to the importance of correcting the optical light curves for the substantial, if not dominant thermal optical emission. This emission is contributed by not just the host galaxy, but more particularly by the Seyfert nucleus which is known to accrete at a high Eddington rate in this class of AGN. Even a conservative correction for these thermal contaminations, as estimated from the optical imaging and SED analysis, has revealed that for two well-observed optical outbursts of the NLSy1 1H 0323+342, the flux doubling time of the optical synchrotron emission is $\leqslant 1$ hour. A more realistic correction could well bring the time scale significantly further down, making it similar to the flux doubling times of the ultra-rapid VHE (> $100 \mathrm{GeV}$ ) flaring events which have been reported for a few blazars, e.g., PKS 2155-304 (Aharonian et al. 2007) and PKS $1222+216$ (Aleksić et al. 2011). Secondly, from the present observations of the NLSy1 $1 \mathrm{H} 0323+342$, it appears that a large radio-loudness parameter may not be an essential condition for the occurrence of strong INOV in radio and $\gamma$-ray NLSy1 galaxies. Thirdly, we caution that estimating the two wellknown parameters which are commonly employed in the context of INOV, namely optical polarization and radio loudness parameter can be challenging in the case of NLSy1s, due to the expected substantial, if not large thermal optical contamination, as well as the marked faintness of their diffuse (i.e., un-boosted) radio components. The interesting 
$1 \mathrm{H} 0323+342$, aperture radius $=2 \times \mathrm{FWHM}$

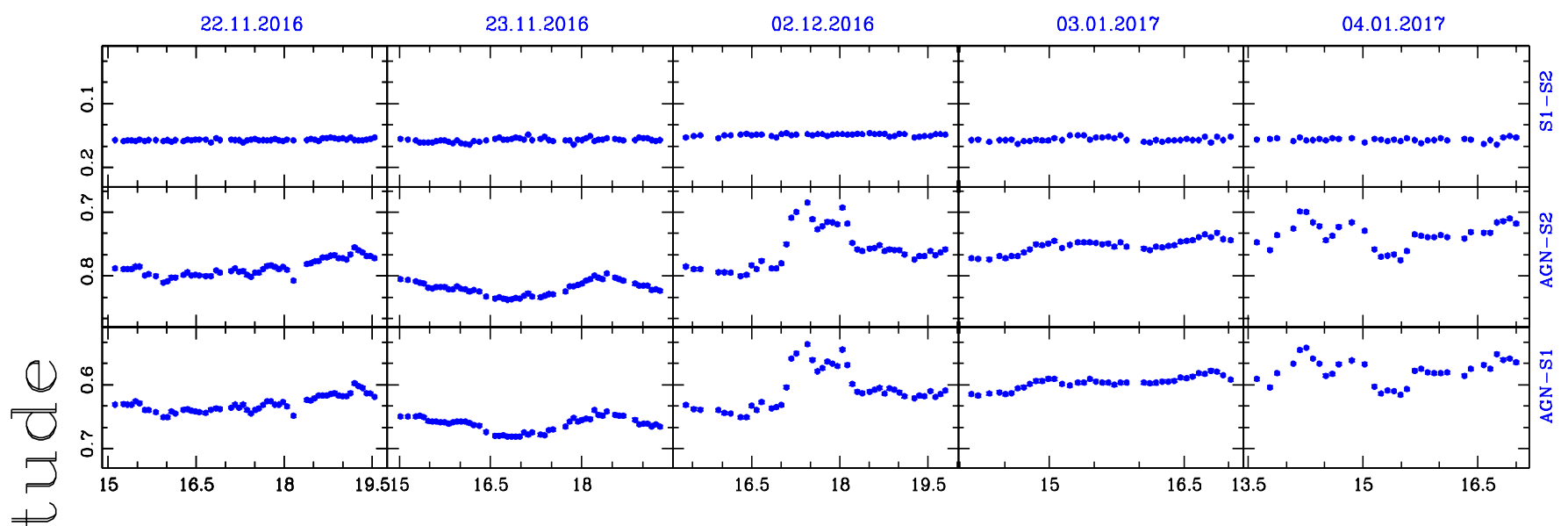

PKS J1222 +0413, aperture radius $=2 x F W H M$

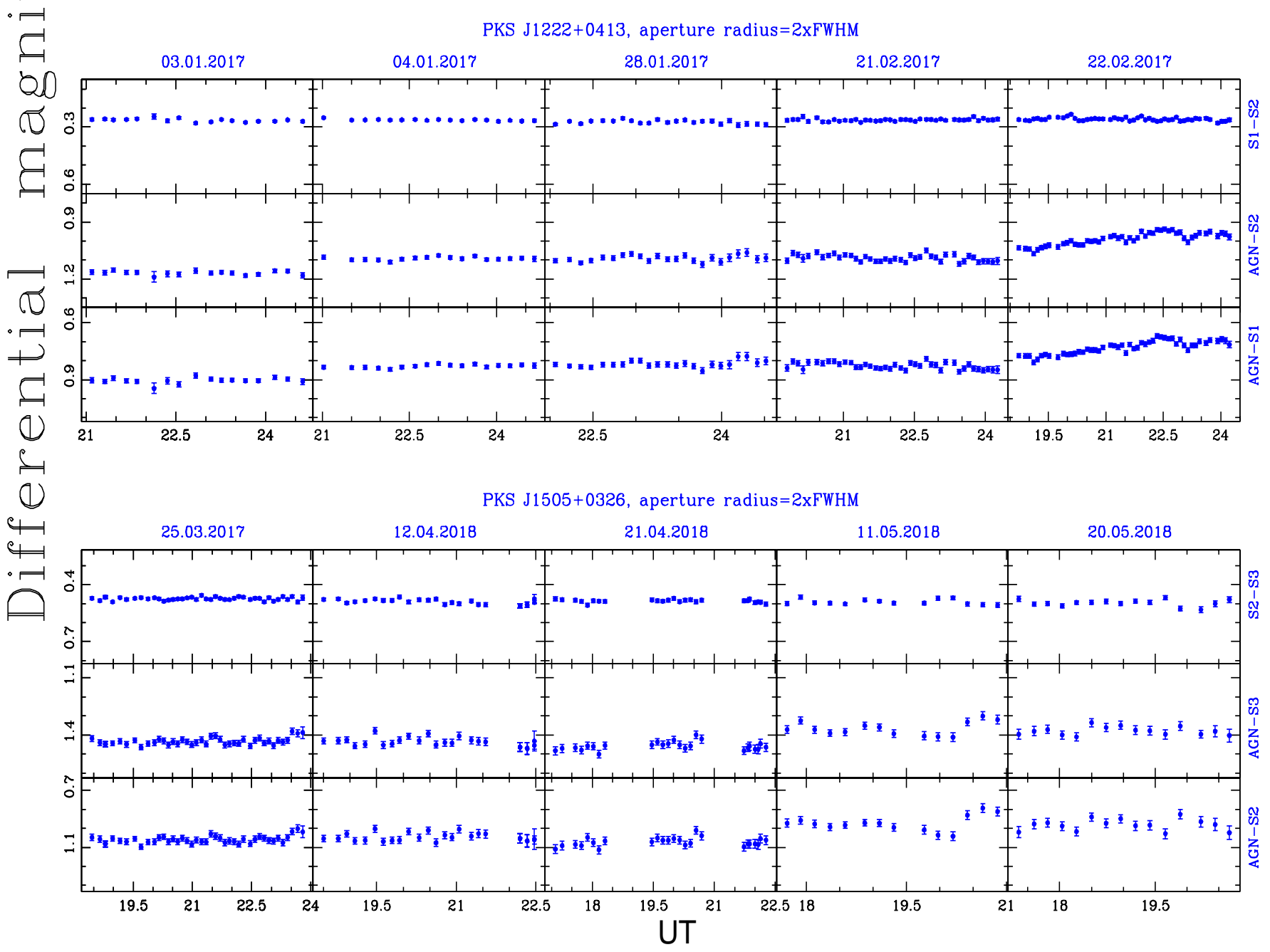

Figure 1. Medium/long - term DLCs of the three $\gamma$-ray NLSy1s. The names of the NLSy1s and their dates of observations are given at the top of the panels. In each panel, the upper DLC is derived using the two non-varying comparison stars, while the lower two DLCs are the 'NLSy1-star' DLCs, as defined in the labels on the right side.

hint emerging from the present study of an admittedly small sample of three $\gamma$-ray detected, radio-loud NLSy1 galaxies is that radio properties like polarization and, perhaps more evidently, the jet's superluminal motion, are likely to serve as more potent diagnostic of INOV in the case of narrow-line Seyfert1 galaxies. 
$1 \mathrm{H} 0323+342$, aperture radius $=6 \mathrm{xFWHM}$

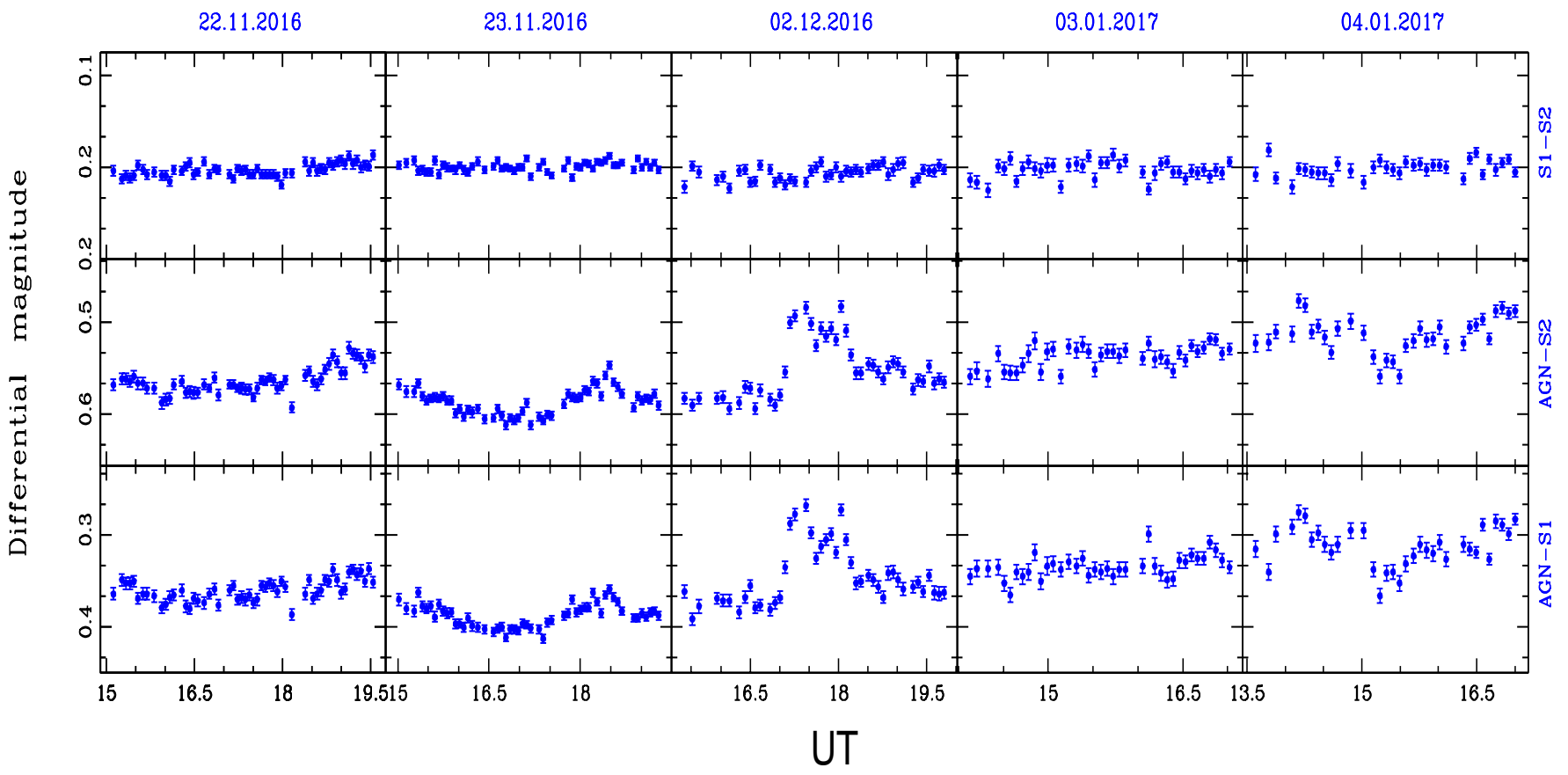

Figure 2. Same as Fig. 1 for $1 \mathrm{H} 0323+342$, but for an aperture radius $=6 \times$ FWHM.

\section{ACKNOWLEDGMENTS}

We thank an anonymous referee for the helpful suggestions.

\section{References}

Abdo A. A. et al., 2010, ApJ, 716, 835

Abdo A. A. et al., 2009a, ApJ, 699, 976

Abdo A. A. et al., 2009b, ApJ, 707, 727

Abdo A. A. et al., 2009c, ApJ, 707, L142

Abdo A. A. et al., 2011, ApJ, 736, 131

Ackermann M. et al., 2014, ApJ, 786, 157

Ackermann M. et al., 2015, ApJ, 810, 14

Adelman-McCarthy J. K. et al., 2006, ApJS, 162, 38

Aharonian F. et al., 2007, ApJ, 664, L71

Aleksić J. et al., 2011, ApJ, 730, L8

Angelakis E. et al., 2015, A\&A, 575, A55

Angelakis E., Kiehlmann S., Myserlis I., Blinov D., Eggen J., Itoh R., Marchili N., Zensus J. A., 2018, ArXiv eprints:1807.02382

Antón S., Browne I. W. A., Marchã M. J., 2008, A\&A, 490, 583

Antonucci R., 2012, Astronomical and Astrophysical Transactions, 27, 557

Bachev R., Strigachev A., Semkov E., 2005, MNRAS, 358, 774

Bagchi J. et al., 2014, ApJ, 788, 174

Bai J. M., Xie G. Z., Li K. H., Zhang X., Liu W. W., 1999, A\&AS, 136, 455

Baldi R. D., Capetti A., Robinson A., Laor A., Behar E., 2016, MNRAS, 458, L69

Barvainis R., Lehár J., Birkinshaw M., Falcke H., Blundell K. M., 2005, ApJ, 618, 108
Begelman M. C., Fabian A. C., Rees M. J., 2008, MNRAS, 384, L19

Berton M., 2018, ArXiv e-prints 1805.08534

Bian W., Zhao Y., 2004, MNRAS, 352, 823

Boller T., Brandt W. N., Fink H., 1996, A\&A, 305, 53

Boroson T. A., 2002, ApJ, 565, 78

Boroson T. A., Green R. F., 1992, ApJS, 80, 109

Boyce P. J. et al., 1998, MNRAS, 298, 121

Britzen S. et al., 2018, MNRAS, 478, 3199

Calderone G., Foschini L., Ghisellini G., Colpi M., Maraschi

L., Tavecchio F., Decarli R., Tagliaferri G., 2011, MNRAS, 413, 2365

Calderone G., Ghisellini G., Colpi M., Dotti M., 2013, MNRAS, 431, 210

Caplar N., Lilly S. J., Trakhtenbrot B., 2017, ApJ, 834, 111

Carini M. T., Miller H. R., Noble J. C., Goodrich B. D., 1992, AJ, 104, 15

Carini M. T., Noble J. C., Taylor R., Culler R., 2007, AJ, 133, 303

Carpenter B., Ojha R., 2013, The Astronomer's Telegram, 5344

Cellone S. A., Romero G. E., Combi J. A., 2000, AJ, 119, 1534

Chen S. et al., 2018, ArXiv e-prints 1801.07234

Chiaberge M., Capetti A., Macchetto F. D., 2005, ApJ, 625, 716

Chiaberge M., Marconi A., 2011, MNRAS, 416, 917

Congiu E. et al., 2017, A\&A, 603, A32

Courvoisier T. J.-L., 1998, A\&A Rev., 9, 1

Coziol R., Andernach H., Torres-Papaqui J. P., Ortega-

Minakata R. A., Moreno del Rio F., 2017, MNRAS, 466, 921

Cracco V., Ciroi S., Berton M., Di Mille F., Foschini L., La

Mura G., Rafanelli P., 2016, MNRAS, 462, 1256

Crenshaw D. M., Kraemer S. B., Gabel J. R., 2003, AJ, 126, 
1H $0323+342,02.12 .2016(4.41 \mathrm{hrs})$ aperture radius $=4 \mathrm{XFWHM}$

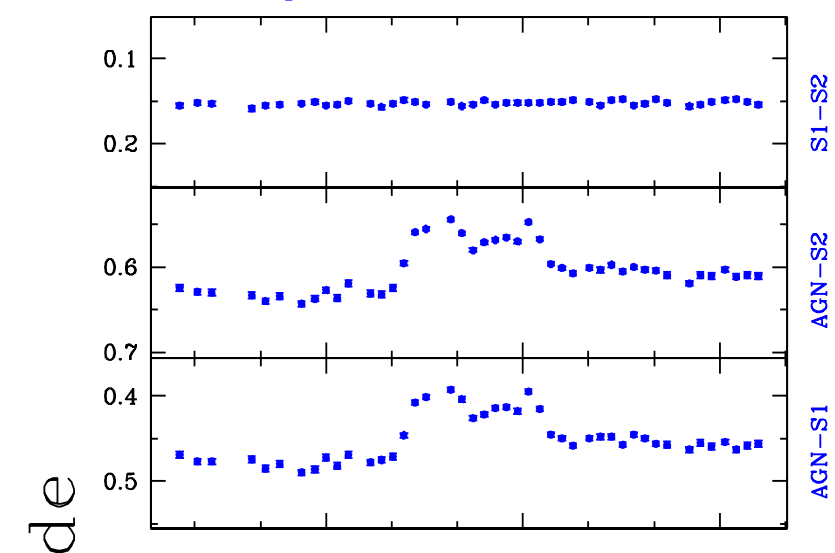

aperture radius $=6 \mathrm{xFWHM}$
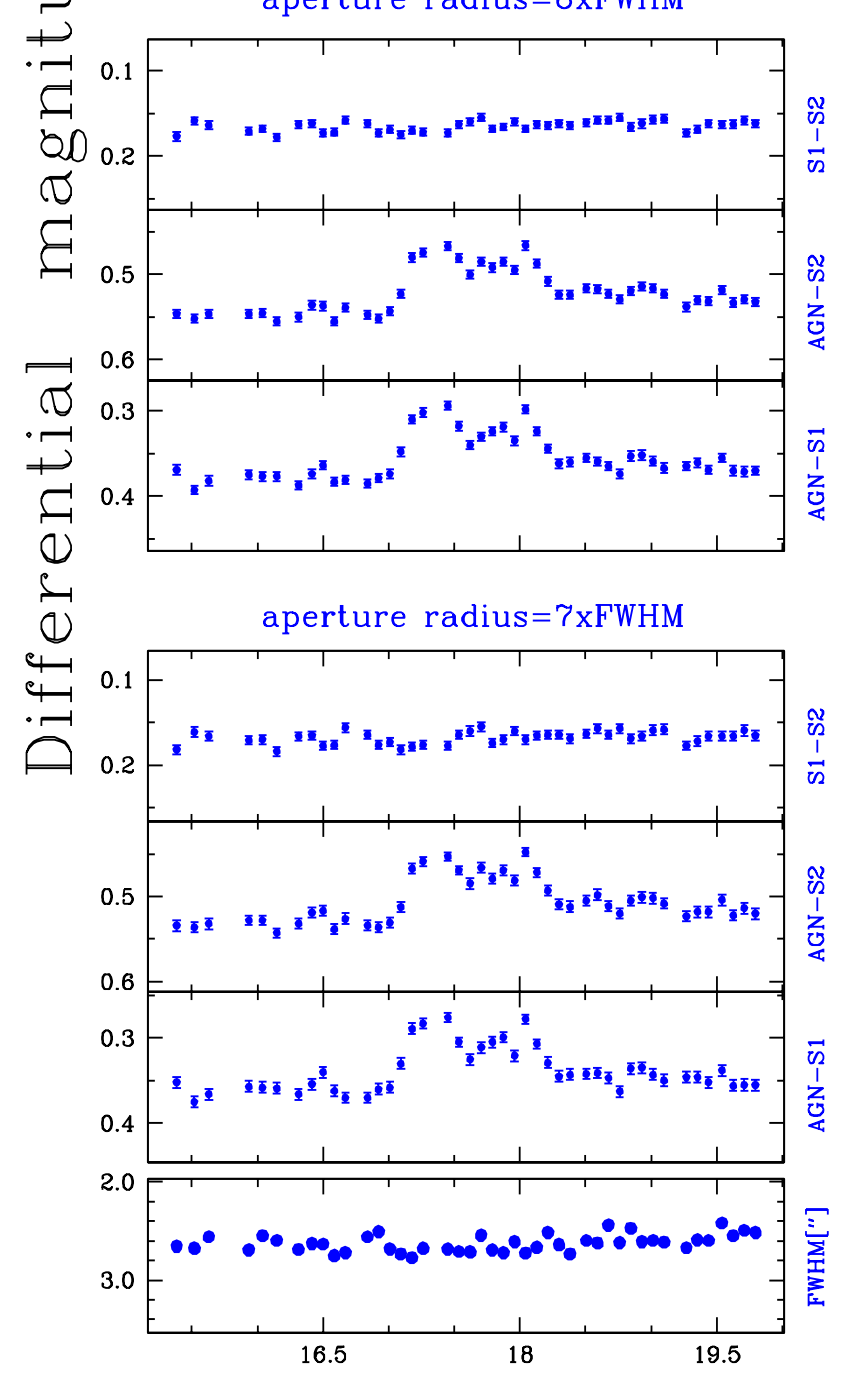

UT

Figure 3. DLCs of $1 \mathrm{H} 0323+342$ derived using three aperture radii, $4 \times \mathrm{FWHM}, 6 \times \mathrm{FWHM}$ and $7 \times \mathrm{FWHM}$ and plotted in the figure from the top to bottom panels, respectively, along with the seeing profile for the session (bottom panel).
1690

Czerny B., Siemiginowska A., Janiuk A., Nikiel-Wroczyński B., Stawarz Ł., 2009, ApJ, 698, 840

D'Ammando F., Ciprini S., 2015, The Astronomer's Telegram, 8447

D'Ammando F. et al., 2013, MNRAS, 433, 952

D'Ammando F. et al., 2016a, MNRAS, 463, 4469

D'Ammando F., Orienti M., Finke J., Larsson J., Giroletti M.,

Raiteri C., 2016b, Galaxies, 4, 11

D'Ammando F. et al., 2012, MNRAS, 426, 317

D'Ammando F. et al., 2015a, MNRAS, 446, 2456

D'Ammando F., Orienti M., Larsson J., Giroletti M., 2015b, MNRAS, 452, 520

de Diego J. A., 2010, AJ, 139, 1269

Decarli R., Dotti M., Fontana M., Haardt F., 2008, MNRAS, 386, L15

Deo R. P., Crenshaw D. M., Kraemer S. B., 2006, AJ, 132, 321

Dermer C. D., Finke J. D., Krug H., Böttcher M., 2009, ApJ, 692, 32

Diamond-Stanic A. M., Rieke G. H., Rigby J. R., 2009, ApJ, 698,623

Doi A., Nagai H., Asada K., Kameno S., Wajima K., Inoue M., 2006, PASJ, 58, 829

Doi A., Nagira H., Kawakatu N., Kino M., Nagai H., Asada K., 2012, ApJ, 760, 41

Drake A. J. et al., 2009, ApJ, 696, 870

Dunlop J. S., McLure R. J., Kukula M. J., Baum S. A., O’Dea C. P., Hughes D. H., 2003, MNRAS, 340, 1095

Eggen J., Miller H. R., Maune J., 2011, in Narrow-Line Seyfert 1 Galaxies and their Place in the Universe, p. 49

Eggen J. R., Miller H. R., Maune J. D., 2013, ApJ, 773, 85

Falcke H., Patnaik A. R., Sherwood W., 1996a, ApJ, 473, L13

Falcke H., Sherwood W., Patnaik A. R., 1996b, ApJ, 471, 106

Fan J. H., Qian B. C., Tao J., 2001, A\&A, 369, 758

Ferrara E. C., Miller H. R., McFarland J. P., Williams A. M., Wilson J. W., Fried R. E., Noble J. C., 2001, in Astronomical Society of the Pacific Conference Series, Vol. 224, Probing the Physics of Active Galactic Nuclei, Peterson B. M., Pogge R. W., Polidan R. S., eds., p. 319

Finke J. D., Dermer C. D., Böttcher M., 2008, ApJ, 686, 181

Foschini L., 2011, in Narrow-Line Seyfert 1 Galaxies and their Place in the Universe, p. 24

Foschini L. et al., 2015, A\&A, 575, A13

Foschini L. et al., 2011, MNRAS, 413, 1671

Fugmann W., 1988, A\&A, 205, 86

Fuhrmann L. et al., 2016, Research in Astronomy and Astrophysics, 16, 176

Garcia A., Sodré L., Jablonski F. J., Terlevich R. J., 1999, MNRAS, 309, 803

Gaskell C. M., 2006, in Astronomical Society of the Pacific Conference Series, Vol. 360, Astronomical Society of the Pacific Conference Series, Gaskell C. M., McHardy I. M., Peterson B. M., Sergeev S. G., eds., p. 111

Ghisellini G., Tavecchio F., 2008, MNRAS, 386, L28

Ghisellini G., Tavecchio F., Chiaberge M., 2005, A\&A, 432, 401

Giannios D., Uzdensky D. A., Begelman M. C., 2009, MNRAS, 395, L29

Giroletti M., Panessa F., 2009, ApJ, 706, L260

Goodrich R. W., Stringfellow G. S., Penrod G. D., Filippenko A. V., 1989, ApJ, 342, 908

Gopal-Krishna, Mangalam A., Wiita P. J., 2008, ApJ, 680, L13 
Gopal-Krishna, Sagar R., Wiita P. J., 1995, MNRAS, 274, 701 Gopal-Krishna, Stalin C. S., Sagar R., Wiita P. J., 2003, ApJ, 586, L25

Gopal-Krishna, Wiita P. J., 2018, Bulletin de la Societe Royale des Sciences de Liege, 87, 281

Gopal-Krishna, Wiita P. J., Altieri B., 1993, A\&A, 271, 89

Goyal A., Gopal-Krishna, Joshi S., Sagar R., Wiita P. J., Anupama G. C., Sahu D. K., 2010, MNRAS, 401, 2622

Goyal A., Gopal-Krishna, Wiita P. J., Anupama G. C., Sahu

D. K., Sagar R., Joshi S., 2012, A\&A, 544, A37

Goyal A., Gopal-Krishna, Paul J. W., Stalin C. S., Sagar R., 2013a, MNRAS, 435, 1300

Goyal A., Mhaskey M., Gopal-Krishna, Wiita P. J., Stalin C. S., Sagar R., 2013b, Journal of Astrophysics and Astronomy, 34, 273

Goyal A. et al., 2017, ArXiv e-prints 1709.04457

Greene J. E., Ho L. C., 2007, ApJ, 667, 131

Grupe D., Beuermann K., Mannheim K., Thomas H.-C., 1999, A\&A, 350, 805

Grupe D., Beuermann K., Thomas H.-C., Mannheim K., Fink H. H., 1998, A\&A, 330, 25

Grupe D., Mathur S., 2004, ApJ, 606, L41

Gupta A. C., Joshi U. C., 2005, A\&A, 440, 855

Heidt J., Wagner S. J., 1996, A\&A, 305, 42

Heidt J., Wagner S. J., Wilhelm-Erkens U., 1997, A\&A, 325, 27

Hovatta T. et al., 2014, MNRAS, 439, 690

Howell S. B., Warnock, III A., Mitchell K. J., 1988, AJ, 95, 247

Hughes P. A., Aller H. D., Aller M. F., 1992, ApJ, 396, 469

Ikejiri Y. et al., 2011, PASJ, 63, 639

Impey C. D., Malkan M. A., Tapia S., 1989, ApJ, 347, 96

Itoh R. et al., 2014, PASJ, 66, 108

Itoh R. et al., 2013, ApJ, 775, L26

Jang M., Miller H. R., 1995, ApJ, 452, 582

Jarvela E., Lähteenmäki A., Lietzen H., Poudel A., Heinämäki P., Einasto M., 2017, A\&A, 606, A9

Jiang N. et al., 2012, ApJ, 759, L31

Jorstad S. G., Marscher A. P., Mattox J. R., Wehrle A. E., Bloom S. D., Yurchenko A. V., 2001, ApJS, 134, 181

Kellermann K. I., Sramek R., Schmidt M., Shaffer D. B., Green R., 1989, AJ, 98, 1195

Kharb P., Lister M. L., Cooper N. J., 2010, ApJ, 710, 764

Klimek E. S., Gaskell C. M., Hedrick C. H., 2004, ApJ, 609, 69

Komossa S., Meerschweinchen J., 2000, A\&A, 354, 411

Komossa S., Voges W., Xu D., Mathur S., Adorf H.-M., Lemson G., Duschl W. J., Grupe D., 2006, AJ, 132, 531

Kshama S. K., Paliya V. S., Stalin C. S., 2017, MNRAS, 466, 2679

Kügler S. D., Nilsson K., Heidt J., Esser J., Schultz T., 2014, A\&A, 569, A95

Kumar P., Gopal-Krishna, Stalin C. S., Chand H., Srianand R., Petitjean P., 2017, MNRAS, 471, 606

Lähteenmäki A., Järvelä E., Ramakrishnan V., Tornikoski M., Tammi J., Vera R. J. C., Chamani W., 2018, A\&A, 614, L1

Laor A., 2000, ApJ, 543, L111

Laor A., Behar E., 2008, MNRAS, 390, 847

Leighly K. M., 1999, ApJS, 125, 297

León Tavares J. et al., 2014, ApJ, 795, 58

Lister M. L. et al., 2016, AJ, 152, 12

Lister M. L., Homan D. C., 2005, AJ, 130, 1389
Lister M. L., Homan D. C., Kadler M., Kellermann K. I., Kovalev Y. Y., Ros E., Savolainen T., Zensus J. A., 2009, ApJ, 696, L22

Liu H., Wang J., Mao Y., Wei J., 2010, ApJ, 715, L113

Liu H., Wu C., Wang J., Wei J., 2016, New A, 44, 51

Mangalam A. V., Wiita P. J., 1993, ApJ, 406, 420

Marconi A., Axon D. J., Maiolino R., Nagao T., Pastorini G.,

Pietrini P., Robinson A., Torricelli G., 2008, ApJ, 678, 693

Marscher A. P., 2014, ApJ, 780, 87

Marscher A. P., Gear W. K., Travis J. P., 1992, in Variability of Blazars, Valtaoja E., Valtonen M., eds., p. 85

Mathur S., 2000, MNRAS, 314, L17

Maune J. D., Eggen J. R., Miller H. R., Marshall K., Readhead A. C. S., Hovatta T., King O., 2014, ApJ, 794, 93

McHardy I. M., Merrifield M. R., Abraham R. G., Crawford C. S., 1994, MNRAS, 268, 681

McLure R. J., Jarvis M. J., 2004, MNRAS, 353, L45

Miller H. R., Carini M. T., Goodrich B. D., 1989, Nature, 337, 627

Miller H. R., Ferrara E. C., McFarland J. P., Wilson J. W., Daya A. B., Fried R. E., 2000, New A Rev., 44, 539

Miller H. R., Noble J. C., 1996, in Astronomical Society of the Pacific Conference Series, Vol. 110, Blazar Continuum Variability, Miller H. R., Webb J. R., Noble J. C., eds., p. 17 Miller P., Rawlings S., Saunders R., 1993, MNRAS, 263, 425

Monet D. G., 1998, in Bulletin of the American Astronomical Society, Vol. 30, American Astronomical Society Meeting Abstracts, p. 1427

Nagao T., Murayama T., Taniguchi Y., 2001, ApJ, 546, 744

Neumann M., Reich W., Fuerst E., Brinkmann W., Reich P., Siebert J., Wielebinski R., Truemper J., 1994, A\&AS, 106, 303

Olguín-Iglesias A. et al., 2016, MNRAS, 460, 3202

Orienti M., D'Ammando F., Giroletti M., for the Fermi-LAT Collaboration, 2012, ArXiv e-prints 1205.0402

Osterbrock D. E., Pogge R. W., 1985, ApJ, 297, 166

Paliya V. S., Ajello M., Rakshit S., Mandal A. K., Stalin C. S., Kaur A., Hartmann D., 2018, ApJ, 853, L2

Paliya V. S., Rajput B., Stalin C. S., Pandey S. B., 2016, ApJ, 819,121

Paliya V. S., Sahayanathan S., Parker M. L., Fabian A. C., Stalin C. S., Anjum A., Pandey S. B., 2014, ApJ, 789, 143

Paliya V. S., Stalin C. S., 2016, ApJ, 820, 52

Paliya V. S., Stalin C. S., Kumar B., Kumar B., Bhatt V. K., Pandey S. B., Yadav R. K. S., 2013, MNRAS, 428, 2450

Pavlidou V. et al., 2014, MNRAS, 442, 1693

Peterson B. M. et al., 2000, ApJ, 542, 161

Pogge R. W., 2000, New A Rev., 44, 381

Pogge R. W., 2011, in Narrow-Line Seyfert 1 Galaxies and their Place in the Universe, p. 2

Pushkarev A. B., Kovalev Y. Y., 2012, A\&A, 544, A34

Pushkarev A. B., Kovalev Y. Y., Lister M. L., Savolainen T., 2009, A\&A, 507, L33

Rafter S. E., Crenshaw D. M., Wiita P. J., 2009, AJ, 137, 42

Rafter S. E., Crenshaw D. M., Wiita P. J., 2011, AJ, 141, 85

Rakshit S., Stalin C. S., Chand H., Zhang X.-G., 2017, ApJS, 229, 39

Ramírez A., de Diego J. A., Dultzin D., González-Pérez J.-N., 2009, AJ, 138, 991

Readhead A. C. S., Taylor G. B., Pearson T. J., Wilkinson P. N., 1996, ApJ, 460, 634

Rokaki E., Collin-Souffrin S., Magnan C., 1993, A\&A, 272, 8 
Romero G. E., Cellone S. A., Combi J. A., 1999, A\&AS, 135, 477

Romero G. E., Cellone S. A., Combi J. A., 2000, A\&A, 360, L47

Ruan J. J. et al., 2012, ApJ, 760, 51

Sagar R., Kumar B., Omar A., Pandey A. K., 2010, in Astronomical Society of India Conference Series, Vol. 1, Astronomical Society of India Conference Series

Sagar R., Stalin C. S., Gopal-Krishna, Wiita P. J., 2004, MNRAS, 348, 176

Sbarrato T., Dotti M., Ghirlanda G., Tavecchio F., 2018, ArXiv e-prints 1805.06459

Sbarrato T., Ghisellini G., Maraschi L., Colpi M., 2012, MNRAS, 421, 1764

Scarpa R., Urry C. M., Padovani P., Calzetti D., O'Dowd M., 2000, ApJ, 544, 258

Shaw M. S. et al., 2012, ApJ, 748, 49

Shen Y., Ho L. C., 2014, nat, 513, 210

Shuder J. M., Osterbrock D. E., 1981, ApJ, 250, 55

Singal K. A., Gopal-Krishna, 1985, MNRAS, 215, 383

Singh V., Chand H., 2018, ArXiv e-prints 1807.01945

Stalin C. S., Gopal-Krishna, Sagar R., Wiita P. J., 2004a, MNRAS, 350, 175

Stalin C. S., Gopal Krishna, Sagar R., Wiita P. J., 2004b, Journal of Astrophysics and Astronomy, 25, 1

Stalin C. S., Gupta A. C., Gopal-Krishna, Wiita P. J., Sagar R., 2005, MNRAS, 356, 607

Stetson P. B., 1987, PASP, 99, 191

Stetson P. B., 1992, in Astronomical Society of the Pacific Conference Series, Vol. 25, Astronomical Data Analysis Software and Systems I, Worrall D. M., Biemesderfer C., Barnes J., eds., p. 297

Sulentic J. W., Zwitter T., Marziani P., Dultzin-Hacyan D., 2000, ApJ, 536, L5

Tadhunter C., 2016, A\&A Rev., 24, 10

Tavecchio F. et al., 2001, ApJ, 554, 725

Ulrich M.-H., Maraschi L., Urry C. M., 1997, ARA\&A, 35, 445

Urry C. M., Padovani P., 1995, PASP, 107, 803

Valtaoja E., Valtaoja L., Efimov I. S., Shakhovskoi N. M., 1990, AJ, 99, 769

Villforth C., Nilsson K., Østensen R., Heidt J., Niemi S.-M., Pforr J., 2009, MNRAS, 397, 1893

Wagner S. J., Witzel A., 1995, ARA\&A, 33, 163

Wang T., Brinkmann W., Bergeron J., 1996, A\&A, 309, 81

Wang T.-G., Zhou H.-Y., Wang J.-X., Lu Y.-J., Lu Y., 2006, ApJ, 645, 856

White R. L., Becker R. H., 1992, ApJS, 79, 331

Wiita P. J., 2006, in Astronomical Society of the Pacific Conference Series, Vol. 350, Blazar Variability Workshop II: Entering the GLAST Era, Miller H. R., Marshall K., Webb J. R., Aller M. F., eds., p. 183

Wright E. L. et al., 2010, AJ, 140, 1868

Xu D., Komossa S., Zhou H., Lu H., Li C., Grupe D., Wang J., Yuan W., 2012, AJ, 143, 83

Yao S., Yuan W., Zhou H., Komossa S., Zhang J., Qiao E., Liu B., 2015, MNRAS, 454, L16

York D. G. et al., 2000, AJ, 120, 1579

Yuan W., Zhou H. Y., Komossa S., Dong X. B., Wang T. G., Lu H. L., Bai J. M., 2008, ApJ, 685, 801

Zensus J. A., 1997, ARA\&A, 35, 607
Zhou H., Wang T., Yuan W., Lu H., Dong X., Wang J., Lu Y., 2006, ApJS, 166, 128

Zhou H. et al., 2007, ApJ, 658, L13

Zhou H.-Y., Wang T.-G., Dong X.-B., Zhou Y.-Y., Li C., 2003, ApJ, 584, 147 